\title{
Anatomical Evidence for the Involvement of the Macaque Ventrolateral Prefrontal Area 12r in Controlling Goal-Directed Actions
}

\author{
Elena Borra, Marzio Gerbella, Stefano Rozzi, and Giuseppe Luppino \\ Department of Neuroscience, University of Parma and Brain Center of Motor and Social Cognition of Italian Institute of Technology, 43100 Parma, Italy
}

\begin{abstract}
The macaque ventrolateral prefrontal (VLPF) area 12r is thought to be involved in higher-order nonspatial information processing. We found that this area is connectionally heterogeneous, and the intermediate part is fully integrated in a cortical network involved in selecting and controlling object-oriented hand and mouth actions. Specifically, intermediate area $12 \mathrm{r}$ displayed dense connections with the caudal half of area $46 \mathrm{v}$ and orbitofrontal areas and relatively strong extraprefrontal connections involving the following: (1) the handand mouth-related ventral premotor area F5 and the anterior intraparietal (AIP) area, jointly involved in visuomotor transformations for grasping; (2) the SII sector that is connected to AIP and F5; (3) a sector of the inferotemporal area TEa/m, primarily corresponding to the sector densely connected to AIP; and (4) the insular and opercular frontal sectors, which are connected to AIP and F5. This connectivity pattern differed markedly from those of the caudal and rostral parts of area 12r. Caudal area 12r displayed dense connections with the caudal part of the VLPF, including oculomotor areas 8/FEF and 45B, relatively weak orbitofrontal connections and extraprefrontal connections limited to the inferotemporal cortex. Rostral area 12r displayed connections mostly with rostral prefrontal and orbitofrontal areas and relatively weaker connections with the fundus and the upper bank of the superior temporal sulcus. The present data suggest that the intermediate part of area $12 \mathrm{r}$ is involved in nonspatial information processing related to object properties and identity, for selecting and controlling goal-directed hand and mouth actions.
\end{abstract}

\section{Introduction}

Area $12 \mathrm{r}$ of the macaque is located in the ventrolateral prefrontal cortex (VLPF) where it lies ventral to area 46 and rostral to area 45A (Carmichael and Price, 1994). This area is the target of projections from higher-order visual areas of the inferotemporal cortex (Webster et al., 1994) and hosts visual neurons tuned specifically to the identity or features of objects (Wilson et al., 1993). Furthermore, lesion studies have highlighted an involvement of this area in behavioral tasks in which information on object identity plays a key role (Passingham, 1975; Mishkin and Manning, 1978; Wang et al., 2000). Together, these data have suggested a role for this prefrontal sector in higher-order aspects of nonspatial information processing (Levy and Goldman-Rakic, 2000; Passingham et al., 2000; Tanji and Hoshi, 2008).

Recent indirect evidence (Borra et al., 2008; Gerbella et al., 2011) showed that this area is connected to the anterior intraparietal (AIP) area and the anterior (F5a) subdivision of the ventral premotor area F5. Because AIP and F5 are two reciprocally con-

\footnotetext{
Received April 7, 2011; revised June 30, 2011; accepted July 5, 2011.

Author contributions: E.B., M.G., S.R., and G.L. designed research; E.B., M.G., S.R., and G.L. performed research; E.B., M.G., S.R., and G.L. analyzed data; E.B. and G.L. wrote the paper.

The work was supported by Ministero dell'Istruzione, dell'Università e della Ricerca Grant PRIN 2008, 2006052343_002, Belgian Science Policy Office Grant IUAP P6/29, and European Commission Grant Cogsystems FP7-250013. The 3D reconstruction software was developed by CRS4 (Pula, Cagliari, Italy).

Correspondence should be addressed to Giuseppe Luppino, Dipartimento di Neuroscienze, Sezione di Fisiologia, Università di Parma, via Volturno 39E, 43100 Parma, Italy. E-mail: luppino@unipr.it.

DOI:10.1523/JNEUROSCI.1745-11.2011

Copyright $\odot 2011$ the authors $\quad 0270-6474 / 11 / 3112351-13 \$ 15.00 / 0$
}

nected hand-related areas forming a parietofrontal circuit playing a crucial role in visuomotor transformations for grasping (Rizzolatti and Luppino, 2001; Grafton, 2010), these data have suggested a role, not previously hypothesized, for area $12 \mathrm{r}$ in the neural mechanisms for selecting and controlling hand actions.

Very little is known about the cortical connectivity of this VLPF sector. Connections with the parietal and premotor cortex have been observed by Barbas (1988) and Saleem et al. (2008) after tracer injections placed in the middle of area $12 \mathrm{r}$ but not by Gerbella et al. (2010) after injections placed caudally in this area. Indeed, the connections of AIP and F5a with area $12 \mathrm{r}$ observed by Borra et al. (2008) and Gerbella et al. (2011) did not involve the entire extent of this area, only the intermediate part. Thus, area $12 \mathrm{r}$ is, possibly, connectionally not homogeneous.

In the present study, we examined the cortical connections of area $12 \mathrm{r}$, based on tracer injections placed at different rostrocaudal levels. The aims were to identify the area $12 \mathrm{r}$ sector connected to AIP and F5 and to gain insight, based on the cortical connectivity pattern, into the possible involvement of this area in the neural circuits for organizing hand actions.

Parts of this paper have been published previously in abstract form (Borra et al., 2010a).

\section{Materials and Methods}

Subjects, surgical procedures, and selection of the injection sites The experiments were performed in four male macaque monkeys (Macaca mulatta), in which neural tracers were injected at different rostrocaudal levels of area 12r. Animal handling as well as surgical and 
experimental procedures complied with the European guidelines (86/609/EEC and 2003/ 65/EC Directives) and Italian laws in force regarding the care and use of laboratory animals and were approved by the Veterinarian Animal Care and Use Committee of the University of Parma and authorized by the Italian Health Ministry.

Under general anesthesia and aseptic conditions, each animal was placed in a stereotaxic apparatus, and an incision was made in the scalp. The skull was trephined to remove the bone overlying the target region, and the dura was opened to expose the VLPF. The choice of the injection sites was based on identified anatomical landmarks, i.e., the inferior arcuate sulcus, the infraprincipal dimple, and the principal sulcus (PS), and using an average architectonic map of the caudal VLPF providing an estimate of the average location of areas $45 \mathrm{~A}$ and $46 \mathrm{v}$, with respect to these landmarks (Gerbella et al., 2007). These data were then used to estimate the location of the dorsal and the caudal borders of area $12 \mathrm{r}$ with areas $46 \mathrm{v}$ and $45 \mathrm{~A}$, respectively (Fig. 1), and to select the anteroposterior level of the injection sites within the VLPF. After the tracer injections were placed, the dural flap was sutured, the bone was replaced, and the superficial tissues were sutured in layers. During surgery, hydration was maintained with saline, and temperature was maintained using a heating pad. Heart rate, blood pressure, respiratory depth, and body temperature were continuously monitored. After recovery from anesthesia, the animals were returned to their home cages and closely monitored. Dexamethasone and prophylactic broad-spectrum antibiotics were administered preoperatively and postoperatively. Furthermore, analgesics were administered intraoperatively and postoperatively.

\section{Tracer injections and histological procedures}

Once the appropriate site was chosen, the retrograde tracers fast blue (FB) $(3 \%$ in distilled water; Dr. Illing Plastics) and diamidino yellow (DY) $(2 \%$ in $0.2 \mathrm{~m}$ phosphate buffer at $\mathrm{pH} 7.2$; Dr Illing Plastics), the mostly anterograde tracer biotinylated dextran amine (BDA) [10,000 molecular weight (MW), 10\% $0.1 \mathrm{M}$ phosphate buffer, $\mathrm{pH} 7.4$; Invitrogen], and the retro-anterograde tracer dextran conjugated with tetramethylrhodamine [Fluoro-Ruby (FR), $10,000 \mathrm{MW}$, or equal mixture of 10,000 and $3000 \mathrm{MW}$ volumes, $10 \% 0.1 \mathrm{M}$ phosphate buffer, $\mathrm{pH} 7.4$; Invitrogen] or with lucifer yellow dextran (LYD) (10,000 MW, 10\% 0.1 M phosphate buffer, pH 7.4; Invitrogen) were slowly pressure-injected through a glass micropipette (tip diameter, $50-100 \mu \mathrm{m}$ ) attached to a 1 or $5 \mu$ l Hamilton microsyringe. Table 1 summarizes the locations of the injections, the injected tracers, and their amounts.

After appropriate survival periods following the injections (28 d for BDA and FR, 12-14 d for FB and DY), each animal was deeply anesthetized with an overdose of sodium thiopental and perfused consecutively with saline, $3.5-4 \%$ paraformaldehyde, and 5\% glycerol, prepared in 0.1 M phosphate buffer, $\mathrm{pH} 7.4$, through the left cardiac ventricle. Each brain was then blocked coronally on a stereotaxic apparatus, removed from the skull, photographed, and placed in 10\% buffered glycerol for $3 \mathrm{~d}$ and $20 \%$ buffered glycerol for $4 \mathrm{~d}$. Finally, each brain was cut frozen into coronal
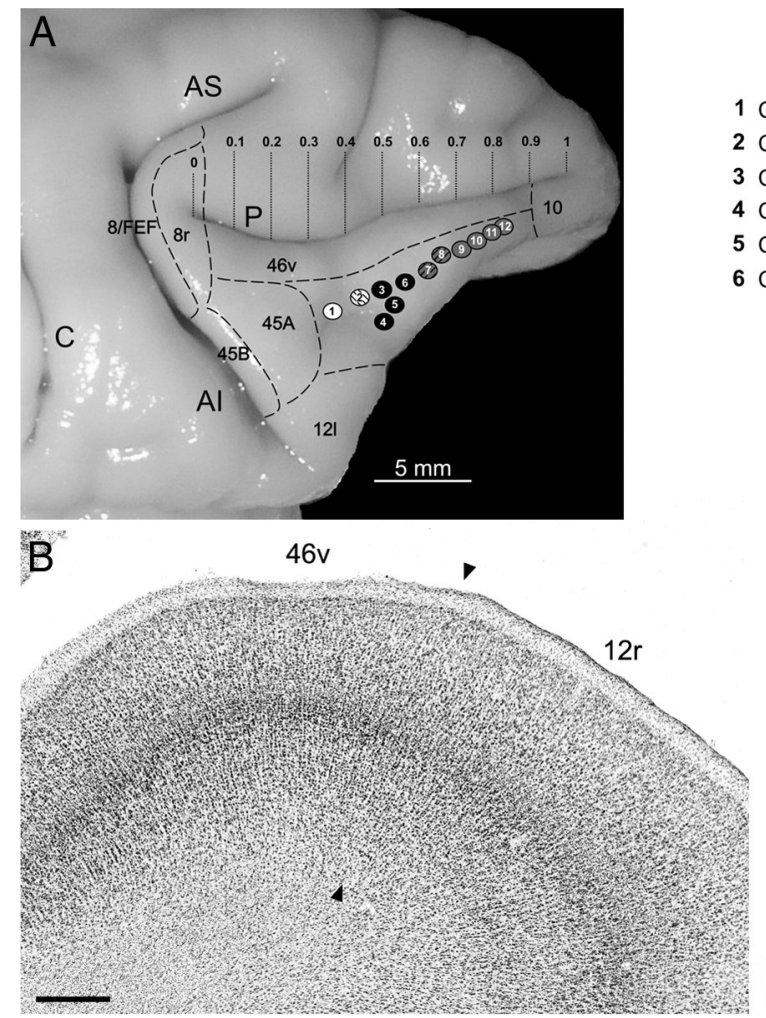
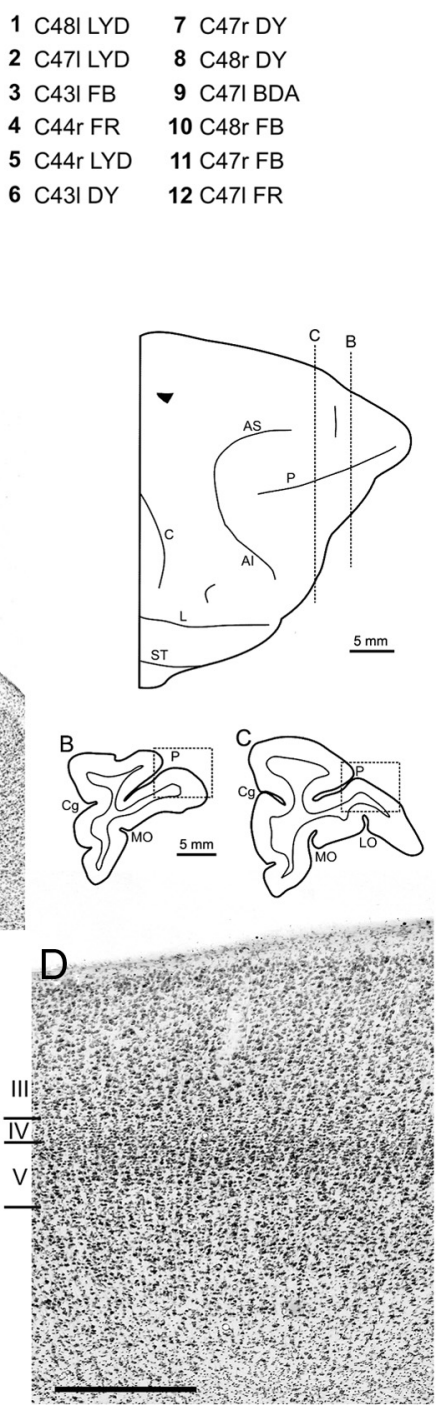

Figure 1. Location of injection sites and cytoarchitecture of area 12r. A, Composite view of all the injection sites mapped on a template hemisphere (Case 44r). Each injection site was reported based on the ratio of its distance from the caudal tip of the PS to to its location in caudal, intermediate, or rostral area 12r, respectively; dashed circles indicate injection sites located in transitional zones. $\boldsymbol{B}, \boldsymbol{C}$, Low-power photomicrographs of Nissl-stained coronal sections through area $12 \mathrm{r}$. Dashed boxes on the sections Arrowheads mark the border of area $12 \mathrm{r}$ with area $46 \mathrm{v}$. D. Higher-magnification view of area $12 \mathrm{r}$, taken from $\boldsymbol{C}$. Scale bars: $\boldsymbol{B}, \boldsymbol{D}, 500$ $\mu \mathrm{m}$ ( $\boldsymbol{B}$ also applies to $\boldsymbol{C}$ ). Al, Inferior arcuate sulcus; $A S$, superior arcuate sulcus; $C$, central sulcus; $C g$, cingulate sulcus; $L$, lateral fissure; LO, lateral orbital sulcus; M0, medial orbital sulcus; P, principal sulcus; ST, superior temporal sulcus.

sections of $60 \mu \mathrm{m}$ thickness. In all cases in which FB or DY was injected, every fifth section was mounted, air dried, and quickly coverslipped for fluorescence microscopy. In Case 47l, one series of each fifth section was processed to visualize BDA (incubation $60 \mathrm{~h}$ ), using a Vectastain $\mathrm{ABC}$ kit (Vector Laboratories) and 3,3' - diaminobenzidine (DAB) as a chromogen. The reaction product was intensified with cobalt chloride and nickel ammonium sulfate. In this same case, two series of each fifth section were processed to visualize FR and BDA, or LYD and BDA, respectively, using the double-labeling protocol described in detail by Gerbella et al. (2010). Briefly, the sections were first processed to visualize BDA, except for a shorter incubation period in the $\mathrm{ABC}$ solution (overnight), and then $\mathrm{BDA}$ was stained brown using $\mathrm{DAB}$. Then, the sections were incubated overnight in avidin-biotin blocking reagent (SP-2001; Vector Laboratories), for $72 \mathrm{~h}$ at $4^{\circ} \mathrm{C}$ in a primary antibody solution of rabbit anti-FR or rabbit anti-LY (1:3000; Invitrogen) in $0.3 \%$ Triton X-100, 5\% normal 
Table 1. Location of injection sites and type and amount of injected tracers

\begin{tabular}{lllclll}
\hline Case & Hemisphere & PS $^{a}$ & Injection core $^{b}$ & Ratio $^{c}$ & Tracer & Amount \\
\hline Case 43 & $\mathrm{L}$ & 19.5 & 9.9 & 0.5 & FB 3\% & $1 \times 0.2 \mu \mathrm{l}$ \\
& $\mathrm{L}$ & & 10.8 & 0.56 & DY 2\% & $1 \times 0.2 \mu \mathrm{l}$ \\
Case 44 & $\mathrm{R}$ & 20.4 & 10.2 & 0.5 & FR 10\% & $1 \times 1 \mu \mathrm{l}$ \\
& $\mathrm{R}$ & & 10.5 & 0.51 & LYD 10\% & $1 \times 1 \mu \mathrm{l}$ \\
Case 47 & $\mathrm{R}$ & 19.0 & 12 & 0.63 & DY 2\% & $1 \times 0.2 \mu \mathrm{l}$ \\
& $\mathrm{R}$ & & 15.3 & 0.8 & FB 3\% & $1 \times 0.2 \mu \mathrm{l}$ \\
& $\mathrm{L}$ & 18.0 & 8.0 & 0.44 & LYD 10\% & $1 \times 1.3 \mu \mathrm{l}$ \\
& $\mathrm{L}$ & & 14.7 & 0.82 & FR 10\% & $1 \times 1 \mu \mathrm{l}$ \\
& $\mathrm{L}$ & & 13.2 & 0.73 & BDA 10\% & $2 \times 1 \mu \mathrm{l}$ \\
Case 48 & $\mathrm{R}$ & 19.5 & 15.0 & 0.77 & FB 3\% & $1 \times 0.2 \mu \mathrm{l}$ \\
& $\mathrm{R}$ & & 12.9 & 0.66 & DY 2\% & $1 \times 0.2 \mu \mathrm{l}$ \\
& $\mathrm{L}$ & 19.5 & 6.9 & 0.35 & LYD 10\% & $1 \times 1.3 \mu \mathrm{l}$ \\
\hline L & & & & &
\end{tabular}

L, Left; R, right.

${ }^{a}$ Length of the PS (in millimeters) in the AP plane.

${ }^{b}$ Distance of the injection site (in millimeters) from the caudal tip of the PS in the AP plane.

'Ratio of the distance of the injection site from the caudal tip of the PS to the length of the PS.

${ }^{d}$ Mix 1:1 of the 3000/10,000 MW.

goat serum in PBS, and $1 \mathrm{~h}$ in biotinylated secondary antibody (1:200; Vector Laboratories) in $0.3 \%$ Triton X-100, $5 \%$ normal goat serum in PBS. Finally, FR or LYD labeling was visualized using the Vectastain ABC kit (Vector Laboratories) and the Vector SG peroxidase substrate kit (SK-4700; Vector Laboratories) as a chromogen. With this procedure, BDA labeling was stained brown, and the FR or the LYD labeling was stained blue in the same tissue sections. In Cases 44r and 481, in which BDA was injected in other cortical areas, the FR and LYD labeling was visualized using the same protocol.

In all cases, one series of each fifth section was stained with the Nissl method $(0.1 \%$ thionin in $0.1 \mathrm{~m}$ acetate buffer, $\mathrm{pH} 3.7)$.

\section{Data analysis}

Injection sites and distribution of retrogradely labeled neurons. The criteria used to define the injection sites and identify FB, DY, BDA, FR, and LYD labeling have been described in previous studies (Luppino et al., 2001, 2003; Rozzi et al., 2006; Gerbella et al., 2010, 2011). All the injection sites used in this study were completely restricted to the cortical gray matter, involving the entire cortical thickness or at least the middle cortical layers. In Case $431 \mathrm{FB}$, the halo of the injection site marginally involved the white matter, but zones I and II, which correspond to the effective area of uptake and transport of fluorescent tracers (Kuypers and Huisman, 1984; Condé, 1987), were confined to the gray matter. Injection sites were attributed to area $12 \mathrm{r}$ with the analysis of adjacent Nissl-stained sections, and the locations of the sites were then reported on a two-dimensional (2D) reconstruction of the injected hemisphere.

Furthermore, to estimate the anteroposterior (AP) position of the injection sites within area $12 \mathrm{r}$, in each case, we measured the distance of the injection site from the caudal tip of the PS along the AP stereotaxic plane and divided the distance by the value of the total AP extent of the PS (Table 1). Because we found that the total length of the PS was quite constant across different cases $(18.9 \pm 0.8 \mathrm{~mm})$, the AP level of each injection site was then reported on a template hemisphere (Case 44r), to obtain a comparative view of the distribution within area 12r (Fig. 1).

The distribution of retrograde (for all tracer injections except BDA) and anterograde (for BDA, FR, and LYD injections) labeling was analyzed in sections every $300 \mu \mathrm{m}$ and plotted in sections every $600 \mu \mathrm{m}$, together with the outer and inner cortical borders, using a computerbased charting system. The distribution of the labeling in the superior temporal sulcus (STS) and in the lateral fissure (LF) was visualized in 2D reconstructions obtained using the same software, as follows (for more details, see Matelli et al., 1998). In each plotted section, the cortical region of interest was unfolded at the level of a virtual line running approximately along the border between layers III and IV. The unfolded sections were then aligned, and the labeling was distributed along the space between the two consecutive plotted sections $(600 \mu \mathrm{m})$. Sections through the STS were aligned to correspond with the fundus and the middle of the floor, and those through the LF were aligned to correspond with the
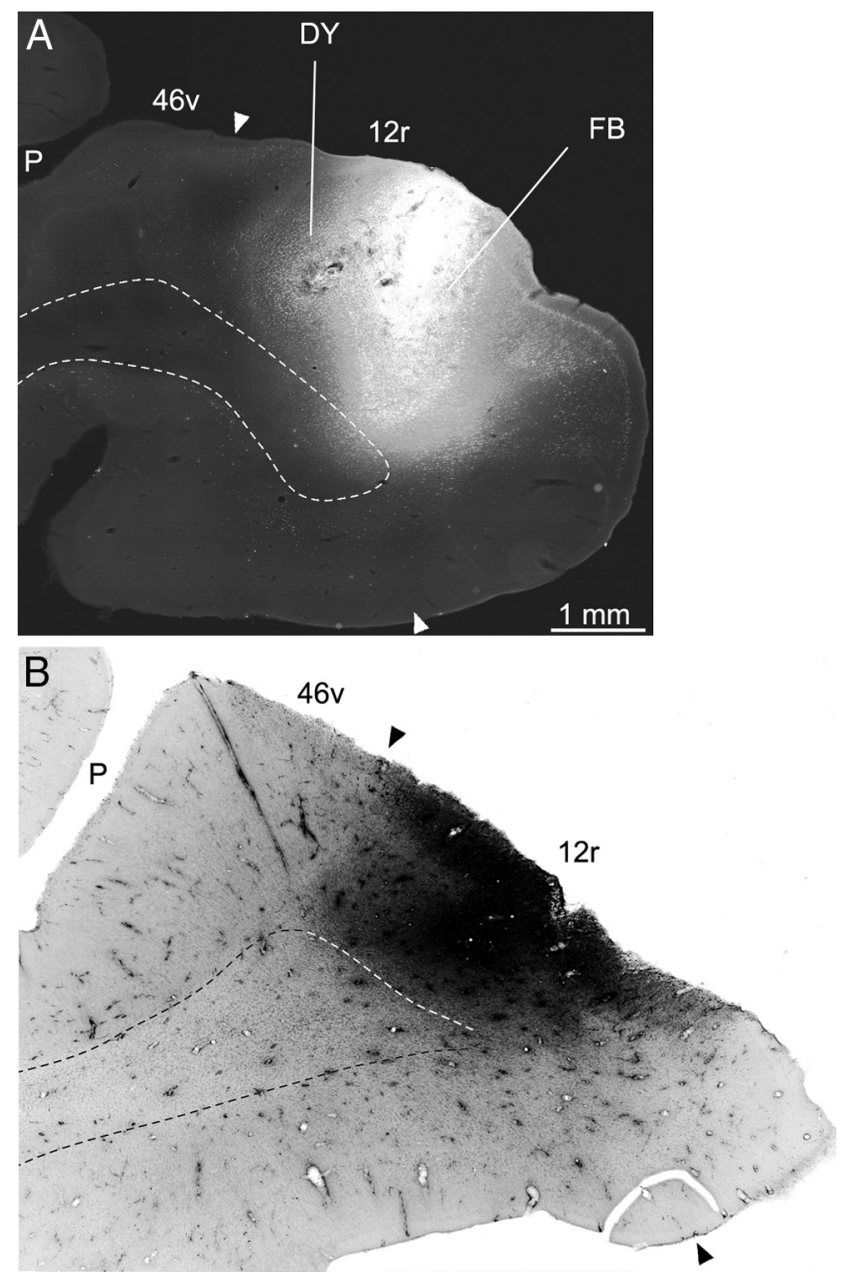

Figure 2. Injection sites in intermediate area 12r. $\boldsymbol{A}, \mathrm{FB}$ and DY injections in Case 43I; $\boldsymbol{B}$, LYD injection in Case 44r. Dashed lines run along the gray/white matter border. Other conventions and abbreviations as in Figure 1.

fundus of the upper bank. Data from individual sections were also imported into the three-dimensional (3D) reconstruction software (Bettio et al., 2001) providing volumetric reconstructions of the monkey brain, including connectional and architectonic data.

Areal attribution of the labeling. The criteria and maps adopted for the areal attribution of the labeling were mostly similar to those adopted in previous studies (Rozzi et al., 2006; Borra et al., 2008; Gerbella et al., 2010, 2011). The prefrontal cortex was subdivided according to Carmichael and Price (1994), except for the caudal VLPF, which was subdivided according to Gerbella et al. (2007). The labeling was attributed to the agranular frontal, cingulate, and opercular frontal areas according to architectonic criteria described previously (Matelli et al., 1985, 1991; Belmalih et al., 2009). The inferotemporal convexity cortex and the STS were subdivided according to Saleem and Tanaka (1996) and Seltzer and Pandya (1978), respectively. In the inferior parietal lobule (IPL), the gyral convexity areas were defined according to Gregoriou et al. (2006) and those of the lateral bank of the intraparietal sulcus (IPS) according to Borra et al. (2008). For the parietal operculum, we matched our data with the functional maps of Fitzgerald et al. (2004).

Quantitative analysis and laminar distribution of the labeling. In all the retrograde tracer injection cases, except for FR, we counted the number of labeled neurons plotted in the ipsilateral hemisphere, beyond the limits of the injected area, in sections at every $600 \mu \mathrm{m}$ interval. Cortical afferents to area $12 \mathrm{r}$ were then expressed in terms of the percentage of labeled neurons found in a given cortical subdivision, with respect to the overall labeling. FR injections were excluded from this analysis because of a dense, nonspecific cell and background staining surrounding the injec- 
tion site, typically observed in our immunostained material (see Gerbella et al., 2010), which extended into the adjacent area 46 and prevented accurate plotting of the retrograde labeling.

Furthermore, to obtain information about the organization of the laminar patterns of the observed connections, the labeling attributed to a given area and reliably observed across different sections and cases was analyzed in sections at every $300 \mu \mathrm{m}$ in terms of the following: (1) laminar distribution of the anterogradely labeled terminals (for BDA, FR, and LYD injections) and (2) percentages of retrogradely labeled neurons located in the superficial (II-III) versus deep (V-VI) layers (s/d ratio). These data were then interpreted, when possible, in light of the two proposed models of laminar patterns of cortical connections: (1) the functional hierarchical model, formalized by Felleman and Van Essen (1991), and (2) the structural model originally described by Barbas and colleagues (Barbas and Rempel-Clower, 1997; Rempel-Clower and Barbas, 2000; Medalla and Barbas, 2006). In the functional hierarchical model, differences in laminar connectivity patterns can be brought back to three main different types: feedforward connections, linking lowerorder with higher-order areas; feedback connections, linking higher-order with lower-order areas; and lateral connections, linking areas located at the same hierarchical level. Feedforward connections terminate mostly in layers IV and lower III and originate mostly from superficial layers (s/d ratio $>70 \%$ ); feedback connections tend to terminate in all layers but layer IV and originate from deep layers ( $\mathrm{s} / \mathrm{d}$ ratio $<30 \%$ ); lateral connections tend to terminate in all layers and originate almost equally from superficial and deep layers. In the structural model, cortical connections show laminar patterns that differ depending on the degree of structural differences (e.g., laminar differentiation, cell density) between the connected areas. Specifically, projections from areas with higher laminar differentiation or cell density to areas with fewer layers or lower neuronal density originate mostly in the upper layers and terminate predominantly in the middle-deep layers. Projections in the opposite direction originate from deep layers and terminate predominantly in the upper layers. Finally, connections between areas of similar structure originate from and terminate in superficial and deep layers.

\section{Results}

\section{Definition of area $12 \mathrm{r}$ and injection site}

\section{locations}

Area 12r, defined by Carmichael and Price (1994) as an architectonic subdivision of Walker's (1940) area 12, is mostly located in the VLPF. In Nissl-stained material, this area displays a layer III relatively homogeneous in cell density with a slight increase in cell size from the dorsal to the ventral part. Layer IV is relatively thin and cell sparse, and layer $\mathrm{V}$ is cell sparse and populated by relatively small pyramids (Fig. $1 B-D$ ). Based on these criteria, we found that area $12 \mathrm{r}$ occupies most of the rostral two-thirds of the VLPF (Fig. 1A). Dorsally, in the proximity of the PS, area $12 \mathrm{r}$ borders area 46 , characterized by a cell-dense layer III almost
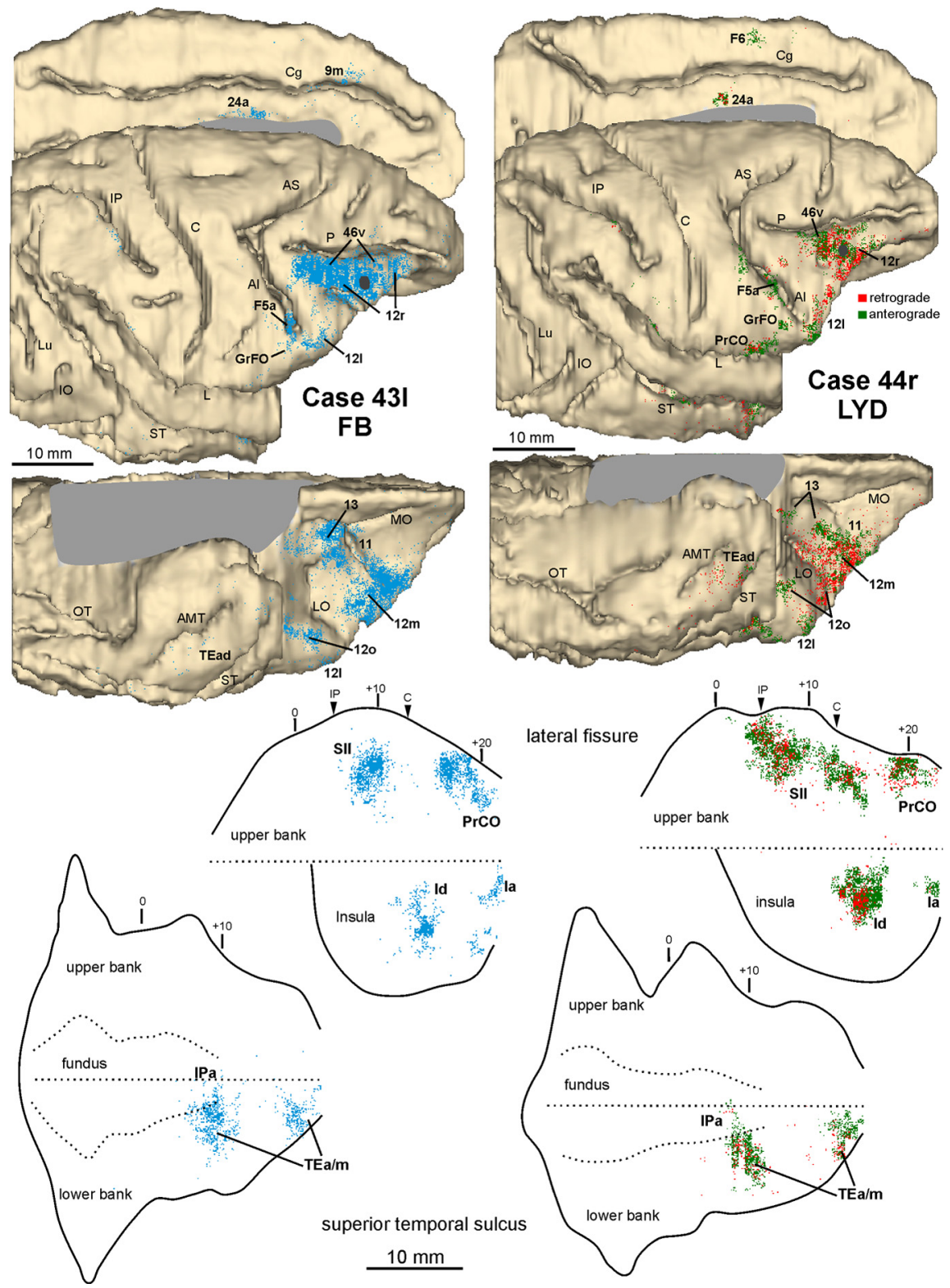

Figure 3. Distribution of the retrograde and retro- (red) anterograde (green) labeling observed after injections in intermediate area $12 r$ in Cases 43I FB and 44r LYD, respectively. The labeling is shown in dorsolateral, medial, and bottom views of the 3D reconstructions of the injected hemispheres (top) and in 2D reconstructions of the LF and of the STS (bottom). For the retrograde labeling, each dot corresponds to one labeled neuron, and for the anterograde labeling, the dot density is proportional to the density of the observed labeled terminals (one dot is equivalent to $\sim 15-25$ labeled terminals). Each 2D reconstruction of the LF was aligned to correspond with the dorsal border of the insula (dashed line). Each 2D reconstruction of the STS was aligned to correspond with the fundus and middle of the floor. The dashed lines indicate the fundus and the upper and lower edges of the floor, and the continuous lines indicate the lips of the sulcus. Short lines mark significant AP levels, and arrowheads mark the AP level of the rostral tip of intraparietal sulcus and of the rostralmost level of the central sulcus. The location of each tracer injection is shown as a dark gray area on the dorsolateral view of the hemisphere. AMT, Anterior middle temporal sulcus; 10 , inferior occipital sulcus; IP, intraparietal sulcus; Lu, lunate sulcus; 0T, occipitotemporal sulcus. Other abbreviations as in Figure 1.

homogeneously populated by relatively small pyramids, a welldeveloped and cell-dense layer IV, and a layer V densely populated by small pyramids. Ventrally, area $12 \mathrm{r}$ extends as far as the lateralmost part of the orbital surface, bordering, from rostral to caudal, areas $111,12 \mathrm{~m}$, and 121 , characterized by a relatively dense layer IV and a sublaminated layer V. Rostrally, in the proximity of the frontal pole, area $12 \mathrm{r}$ borders area 10 , characterized by a dense layer IV and by vertical and horizontal cross striations of cells in layer III and V. Caudally, it borders area 45A, characterized by an evident increase in cell size from the upper to the lower part of 


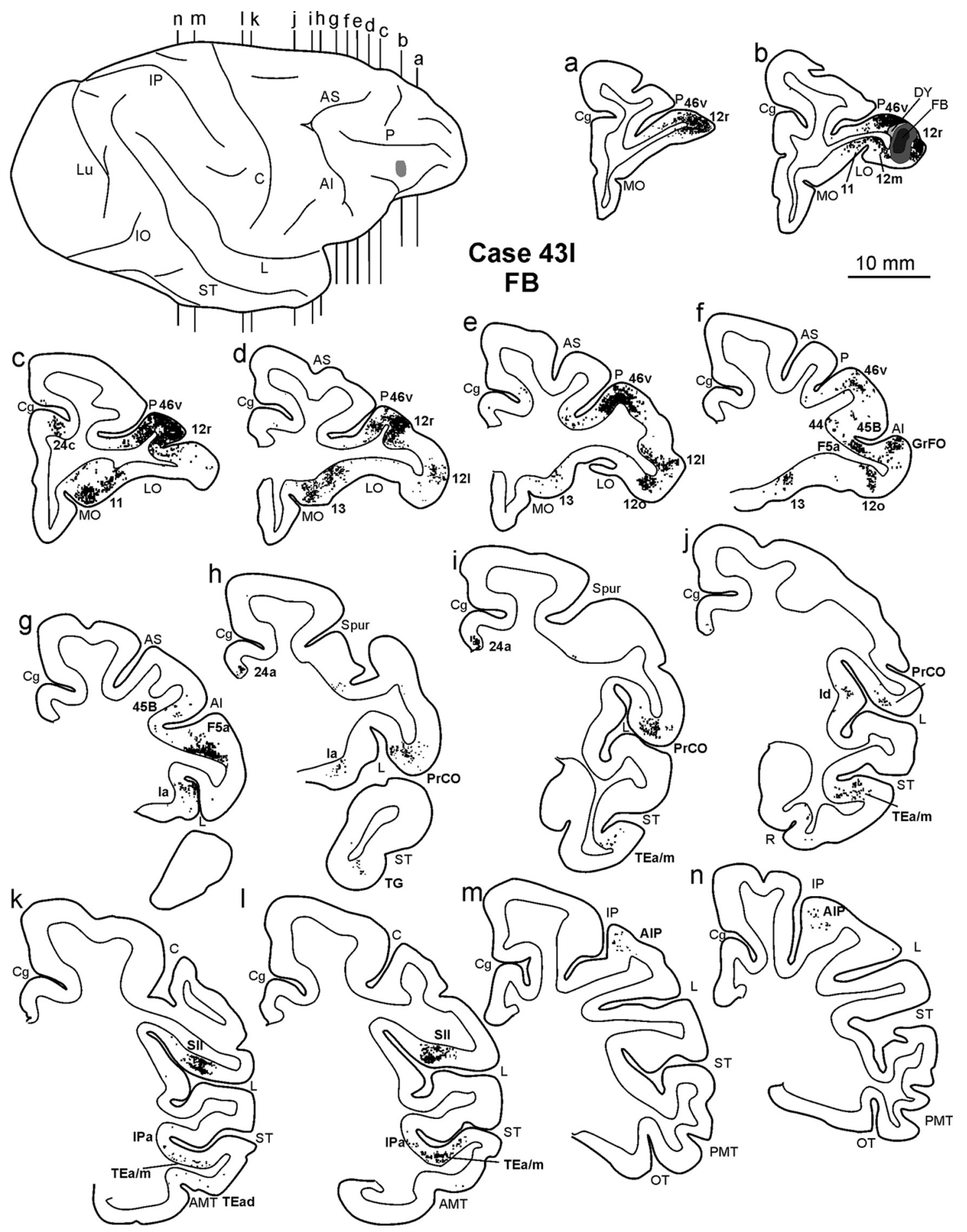

Figure 4. Distribution of the retrograde labeling observed in Case 43I FB, shown in drawing of coronal sections. Sections are shown in a rostral to caudal order (a-n). The dorsolateral view of the injected hemisphere in the top left part shows the levels at which the sections were taken and the location of the injection site. Conventions and abbreviations as in Figures 1 and 3.

layer III, a well-developed layer IV and a relatively dense layer V populated by small pyramids. As described by Petrides and Pandya (2002) and Gerbella et al. (2007), this border corresponds with the infraprincipal dimple, when present.

The injection sites presented in this study were all entirely located within area $12 \mathrm{r}$, at different rostrocaudal and mediolateral levels (Figs. 1 A, 2), involving virtually the entire area. As will be shown later, the results provided evidence of two distinct connectivity patterns displayed by different parts of area $12 \mathrm{r}$. One was observed after injections were placed in the middle of area $12 \mathrm{r}$ (Fig. $1 A$, black circles), i.e., at $\sim 9-12 \mathrm{~mm}$ rostral to the caudal tip of the PS, and the other after injections were placed in the rostral part (Fig. $1 A$, light gray circles), i.e., at $\sim 14-16 \mathrm{~mm}$ rostral to the caudal tip of the PS. These two connectionally distinct parts of area $12 \mathrm{r}$ will be designated here as intermediate and rostral 12r, respectively. Furthermore, the results from Case 481 (Fig. $1 \mathrm{~A}$, white circle), together with our previously published data (Gerbella et al., 2010), showed an additional dis- 


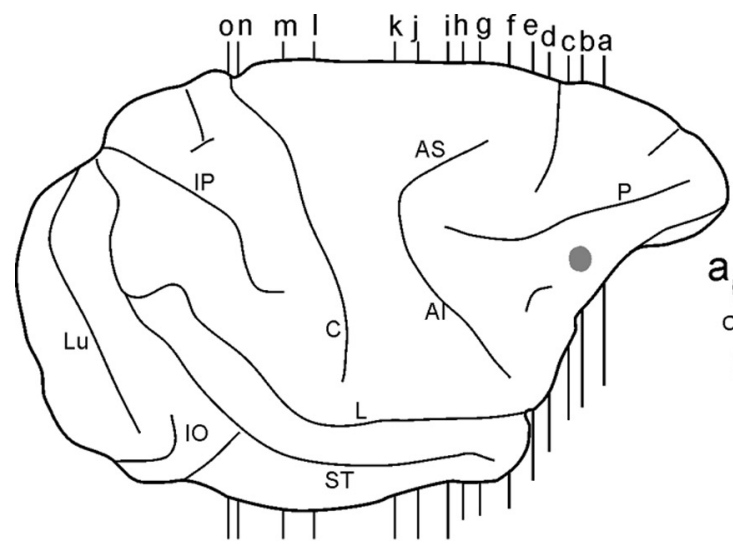

\section{Case 44r} LYD
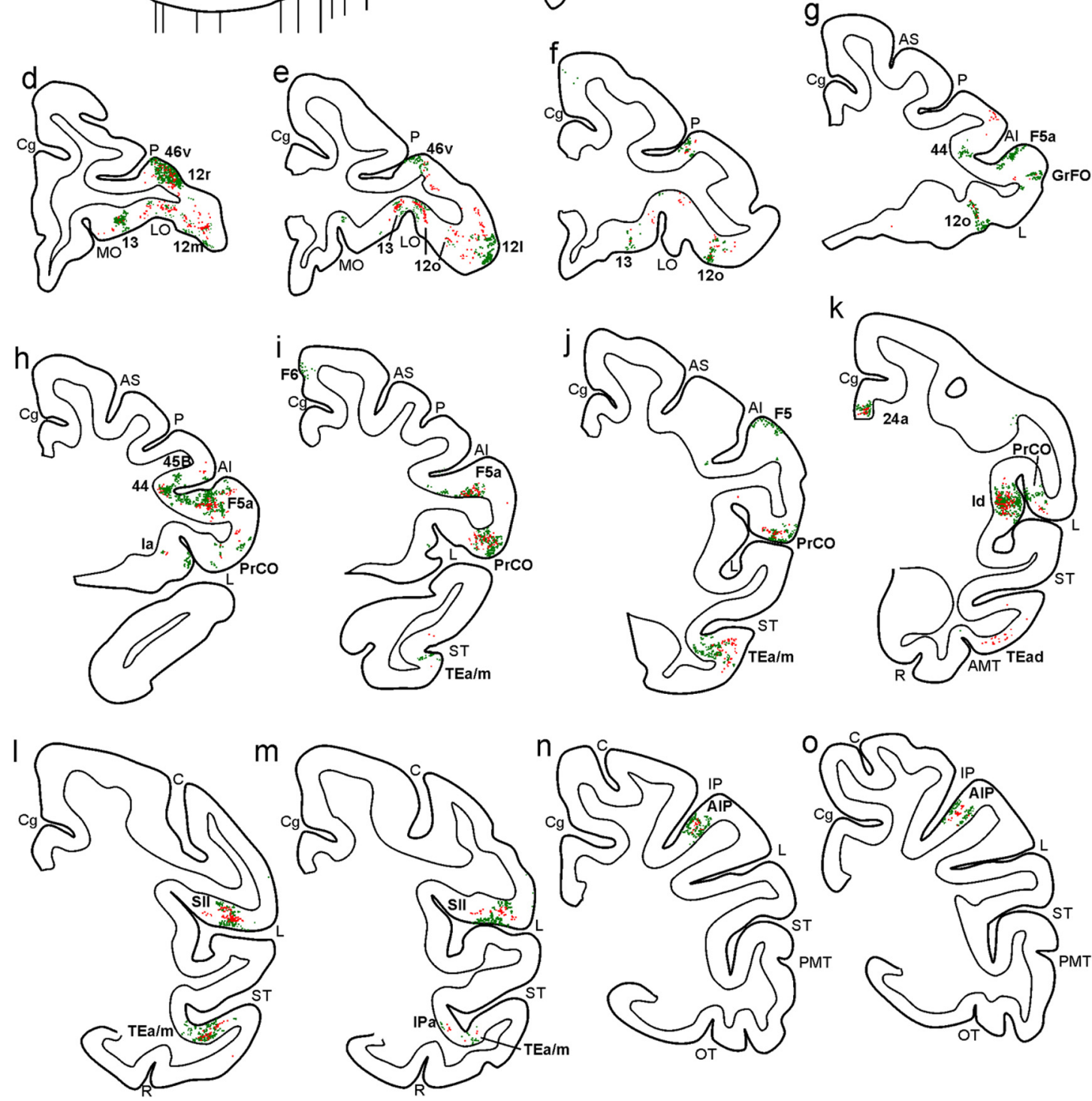

Figure 5. Distribution of the retrograde (red) and anterograde (green) labeling observed in Case 44r LYD, shown in drawing of coronal sections. Sections are shown in a rostral to caudal order $(\boldsymbol{a}-\mathbf{0})$. The dorsolateral view of the injected hemisphere in the top left part shows the levels at which the sections were taken and the location of the injection site. Conventions and abbreviations as in Figures 1 and 3 .

tinct connectivity pattern, attributable to the caudal part of area $12 \mathrm{r}$ (caudal 12r).

\section{Connections of intermediate area $12 \mathrm{r}$}

Four tracer injections (Case 431, FB and DY; Case 44r, FR and LYD) were placed in intermediate area $12 \mathrm{r}$; they involved the entire mediolateral extent. The results from these injections dis- played a connectivity pattern quite consistent across different cases; two (Cases 431 FB and 44r LYD) are shown in Figures 3-5. The percentage distribution of the retrograde labeling observed in Cases 43l, FB and DY, and 44r LYD is shown in Table 2.

In the prefrontal cortex (Fig. 3), the densest labeling, with some quantitative variability across cases, was found in the ventral part of area $46(46 \mathrm{v})$, mostly in the caudal half, along the 


\begin{tabular}{|c|c|c|c|c|c|c|c|}
\hline \multirow[b]{2}{*}{$\begin{array}{l}\text { Injected area } \\
\text { Case }\end{array}$} & \multicolumn{4}{|c|}{ Intermediate $12 r$} & \multicolumn{3}{|c|}{ Rostral 12r } \\
\hline & $\begin{array}{l}431 \mathrm{FB} \\
(\%)\end{array}$ & $\begin{array}{l}43 I \text { DY } \\
(\%)\end{array}$ & $\begin{array}{l}\text { 44r LYD } \\
(\%)\end{array}$ & $\begin{array}{l}\text { Mean } \\
(\%)\end{array}$ & $\begin{array}{l}47 \mathrm{rFB} \\
(\%)\end{array}$ & $\begin{array}{l}48 \mathrm{r} F \mathrm{~B} \\
(\%)\end{array}$ & $\begin{array}{l}\text { Mean } \\
(\%)\end{array}$ \\
\hline \multicolumn{8}{|l|}{ Lateral prefrontal } \\
\hline 10 & - & - & - & - & 10.3 & 11.2 & 10.8 \\
\hline 9 & + & - & - & + & 2.6 & 1.1 & 1.9 \\
\hline $45 \mathrm{~A}$ & + & 1.5 & + & + & 1.9 & - & + \\
\hline $45 B$ & 1.1 & + & + & + & + & + & + \\
\hline $46 v$ & 23.2 & 32.6 & 16.0 & 23.9 & 18.9 & 24.0 & 21.5 \\
\hline $46 \mathrm{~d}$ & - & - & - & - & 6.8 & - & 3.4 \\
\hline 121 & 5.6 & 5.3 & 5.8 & 5.6 & 13.7 & 8.3 & 11.0 \\
\hline \multicolumn{8}{|l|}{ Orbitofrontal } \\
\hline 11 & 8.7 & 11.0 & 10.6 & 10.1 & 16.3 & 19.3 & 17.8 \\
\hline $12 \mathrm{~m}$ & 8.4 & 4.3 & 6.0 & 6.2 & 9.7 & 5.6 & 7.7 \\
\hline 120 & 5.3 & 2.7 & 4.0 & 4.0 & 2.2 & 6.3 & 4.3 \\
\hline 13 & 9.5 & 10.9 & 5.4 & 8.6 & 3.2 & 9.3 & 6.3 \\
\hline 14 & - & - & - & - & 2.7 & 1.2 & 1.9 \\
\hline Total prefrontal & 62.3 & 69.1 & 49.1 & 60.2 & 89.2 & 87.0 & 88.1 \\
\hline \multicolumn{8}{|l|}{ Caudal frontal } \\
\hline PrCO & 5.8 & 5.4 & 10.4 & 7.2 & - & - & - \\
\hline GrFO & 1.8 & 1.1 & + & 1.2 & - & - & - \\
\hline 44 & + & + & 1.1 & + & - & - & - \\
\hline F5 & 9.3 & 7.8 & 5.7 & 7.6 & - & - & - \\
\hline \multicolumn{8}{|l|}{ Temporal } \\
\hline IT convex & + & - & 1.9 & + & + & - & + \\
\hline $\mathrm{TEa} / \mathrm{m}$ & 4.4 & 2.1 & 6.5 & 4.3 & + & - & + \\
\hline $\mathrm{IPa}$ & 1.2 & + & + & + & 1.7 & 4.7 & 3.2 \\
\hline STP & - & - & - & - & 2.3 & 3.0 & 2.7 \\
\hline \multicolumn{8}{|l|}{ Parietal } \\
\hline SII & 5.3 & 7.5 & 10.7 & 7.8 & - & - & - \\
\hline AIP & 1.5 & 1.7 & 2.8 & 2.0 & - & - & - \\
\hline 24 & 2.1 & + & 1.2 & 1.4 & 3.4 & 1.9 & 2.7 \\
\hline Insula & 5.3 & 3.6 & 9.6 & 6.2 & + & 2.4 & 1.3 \\
\hline Other & - & - & - & - & 1.4 & - & + \\
\hline Total cells $(n)$ & 20,076 & 3836 & 3870 & & 17,534 & 11,244 & \\
\hline
\end{tabular}

+ , Labeling $<1 \%$; - no labeling.

shoulder and the lateral part of the bank of the PS (Figs. 4a-f, $5 a-f$ ); weaker labeling involved area $12 \mathrm{l}$. A few clusters of marked cells were located in areas $45 \mathrm{~A}$ and $45 \mathrm{~B}$, and some labeling was inconsistently observed in area $9 \mathrm{~m}$. In the orbitofrontal cortex (Fig. 3), there were dense connections, almost equally involving areas 11 (mostly the lateral part), $12 \mathrm{~m}$, and $12 \mathrm{o}$ and the medial and lateral subdivisions of area 13 (Figs. $4 b-f, 5 a-g$ ).

A high proportion of marked cells was located outside the prefrontal cortex (Fig. 3; Table 2). In the ventral premotor cortex, there were dense connections with area F5a, in the anterior part of the postarcuate bank (Figs. $4 f, g, 5 g-i$ ). Clusters of marked cells were also located in the adjacent fundal dysgranular area 44 . Dense connections were also observed with the dysgranular frontal opercular area PrCO (Figs. $4 h-j, 5 h-k$ ) and a few marked cells more rostrally, in the granular frontal opercular area GrFO. In the parietal cortex, dense labeling was located in the parietal operculum, mostly at an AP level between that of the tip of IPS and that of the central sulcus (Figs. $3,4 k, l, 5 l, m$ ), in the location of the SII region. Specifically, comparison with functional maps published by Fitzgerald et al. (2004) suggests that this labeling likely involves the hand representation and, especially in Case 44r LYD, also the face/mouth representations of the SII region. A weaker connection was consistently observed with AIP (Figs. 4m,n, $5 n, o)$. In the temporal cortex, two densely labeled zones were observed in the lower bank of the STS, in area TEa/m (Fig. 3). One was located very rostrally, the other more caudally, centered at an AP stereotaxic level of approximately +10 , slightly extending in the fundal area IPa (Figs. $4 i-l, 5 i, j, l, m$ ). Sparse labeling was ob- served in the inferotemporal convexity cortex. Finally, there was a consistent connection with a restricted zone of the dysgranular insula (Figs. $4 j, 5 k$ ) and a relatively weaker one with the agranular cingulate area $24 \mathrm{a}$ (Fig. 3).

The laminar distribution of the retrograde and anterograde labeling in the various areas connected to intermediate area $12 \mathrm{r}$ fell within three main general patterns. One pattern, characterized by labeled cells and terminals almost equally distributed in the superficial versus deep cortical layers, was observed in all labeled VLPF areas, in $\mathrm{PrCO}$, and in rostral TEa $/ \mathrm{m}$. This pattern, in Felleman and Van Essen's (1991) model, is the one expected for connections between areas located at a similar hierarchical level and, in Barbas and Rempel-Clower's (1997) structural model, is the one expected for the connections for areas of similar structure. A similar laminar pattern was observed in area F5a (Fig. 6D, G). A second pattern, characterized by labeled cells and terminals considerably denser in the deep layers $(\mathrm{s} / \mathrm{d}$ ratio $<30 \%$ ), was observed in all the orbitofrontal labeled areas (Fig. $6 \mathrm{~A}$ ) and in area 24a; a similar tendency was observed in the dysgranular insula. This pattern, for which Felleman and Van Essen's model does not provide, corresponds to that expected by the structural model for the connections of more differentiated VLPF areas with less differentiated orbitofrontal areas. Finally, a third pattern, characterized by labeled cells considerably denser in the superficial layers ( $\mathrm{s} / \mathrm{d}$ ratio $>70 \%)$ and labeled terminals richer in layer VI and even more in layer I, was observed in the more caudal part of the labeled TEa/m sector (Fig. $6 B, E$ ), in the SII region (Fig. 6C,F), and in AIP. This pattern closely resembled the feedback pattern of the functional hierarchical and the structural models, thus suggesting that area $12 \mathrm{r}$ is at a higher hierarchical level or less differentiated than these connected temporal and parietal areas.

\section{Connections of rostral area $12 \mathrm{r}$}

Four tracer injections (Case 47r FB, 47l, FR and BDA, 48r FB), placed in rostral area $12 \mathrm{r}$ (Fig. 1), displayed a connectivity pattern quite consistent across different cases and markedly different from that observed for intermediate area 12r. The results for two injections (Case 47r FB and 48r FB) are shown in Figures 7 and 8 and in Table 2. A very similar distribution of retro-anterograde or anterograde labeling was observed in Case 47l, FR and BDA.

After the injections were placed in rostral area 12r, almost all the labeling was observed in the prefrontal cortex (Fig. 7; Table 2). Very dense labeling was observed rostrally in area 10 and in the dorsally adjacent rostral part of area 46v (Fig. $8 a-c$ ). Dense connections were also observed caudally with area 121 and weaker ones involved area 45B and, with some variability across cases, area $45 \mathrm{~A}$ (Fig. $8 d-f$ ). Additional labeling was located in area 9. Except for Case 48r FB, clusters of labeled cells or labeled terminals were observed in the fundus of the PS and in the rostral part of dorsal area 46. In the orbitofrontal cortex, as for intermediate area $12 \mathrm{r}$, dense connections were observed with areas 11 (mostly the lateral part), $12 \mathrm{~m}$, and $12 \mathrm{o}$ and the medial and lateral subdivisions of area 13 (Fig. $8 b-f$ ). However, a consistent connection was also observed with area 14 (Fig. 8d,e). Outside the prefrontal cortex, relatively weak connections were consistently observed with the rostral part of the upper bank of the STS and the fundal area IPa, and clusters of labeled cells were found more caudally in the rostral part of the superior temporal polysensory area (STP) (Fig. 8g-i). Finally, a relatively weak connection was observed with area 24 a (Fig. $8 h, i$ ).

After the injections were placed in rostral $12 \mathrm{r}$, the laminar distribution of the retrograde and anterograde labeling in the 
various connected VLPF and orbitofrontal areas was comparable with that observed after the injections were placed in intermediate 12r. In area IPa and in the dorsal bank of the STS, the laminar distribution was similar to that observed in the more caudal part of the TEa/m sector after the intermediate $12 \mathrm{r}$ injections (feedback pattern).

\section{Connections of caudal 12r}

In Case 481 LYD, the injection site, placed at $\sim 7 \mathrm{~mm}$ rostral to the caudal tip of the PS, replicated very well the results observed by Gerbella et al. (2010) after two tracer injections (Cases 26l FB and 39r DY) placed in the caudalmost part of area 12r. These data clearly showed that the connectivity pattern of caudal $12 \mathrm{r}$ is markedly different from that of intermediate $12 \mathrm{r}$. Because after this LYD injection the retrograde labeling was relatively poor, the results will be described here only in terms of the qualitative distribution of retro-anterograde labeling in Figure 9 (for quantitative analysis of the caudal 12 r connections, see Gerbella et al., 2010, their Table 2).

In the prefrontal cortex, the retroanterograde labeling, almost equally distributed in the superficial versus deep cortical layers, was very dense in the caudal VLPF areas 45A, 45B, and $12 \mathrm{l}$ and less dense in area $8 / \mathrm{FEF}$ and the caudal part of area $46 \mathrm{v}$ (Fig. $9 c-f$ ). Additional labeling was observed in the rostral part of area $46 \mathrm{v}$ (Fig. 9a). In the orbitofrontal cortex, the retro-anterograde labeling, mostly concentrated in the deep cortical layers, was relatively dense only in area 120 and less dense in areas $12 \mathrm{~m}$ and 13 (Fig. $9 c, d$ ). Some labeling was also observed in the rostral part of the dorsal premotor cortex, corresponding to the supplementary eye field (SEF) (Fig. $9 c, d$ ). The only significantly labeled sector outside the frontal lobe was the inferotemporal cortex, in which the labeling was mostly concentrated in the rostral and intermediate parts of area $\mathrm{TEa} / \mathrm{m}$, in area IPa, and in areas TEad and TEpd (Fig. $9 h-j$ ). In the intermediate part of area $\mathrm{TEa} / \mathrm{m}$, the labeling occupied a relatively large sector that extended more caudally than that labeled after the injections in intermediate area 12r. In all these cortical sectors, the labeled cells were denser in the superficial layers, and the labeled terminals were denser in layer VI and even more in layer I.

\section{“Transitional” injections}

The injection sites in Cases 47r DY and 48r DY, which were placed at $\sim 13 \mathrm{~mm}$ rostral to the caudal tip of the PS, showed a distribution of retrograde labeling compatible with a transition between the connectivity patterns displayed by the intermediate and rostral 12r. Specifically, in both cases, the distribution of the labeling in the prefrontal cortex was very similar to that observed for intermediate area $12 \mathrm{r}$, although in area $46 \mathrm{v}$ the labeling tended to extend in the rostral part. However, in the parietal and temporal cortices, labeling was virtually absent or very weak, re-
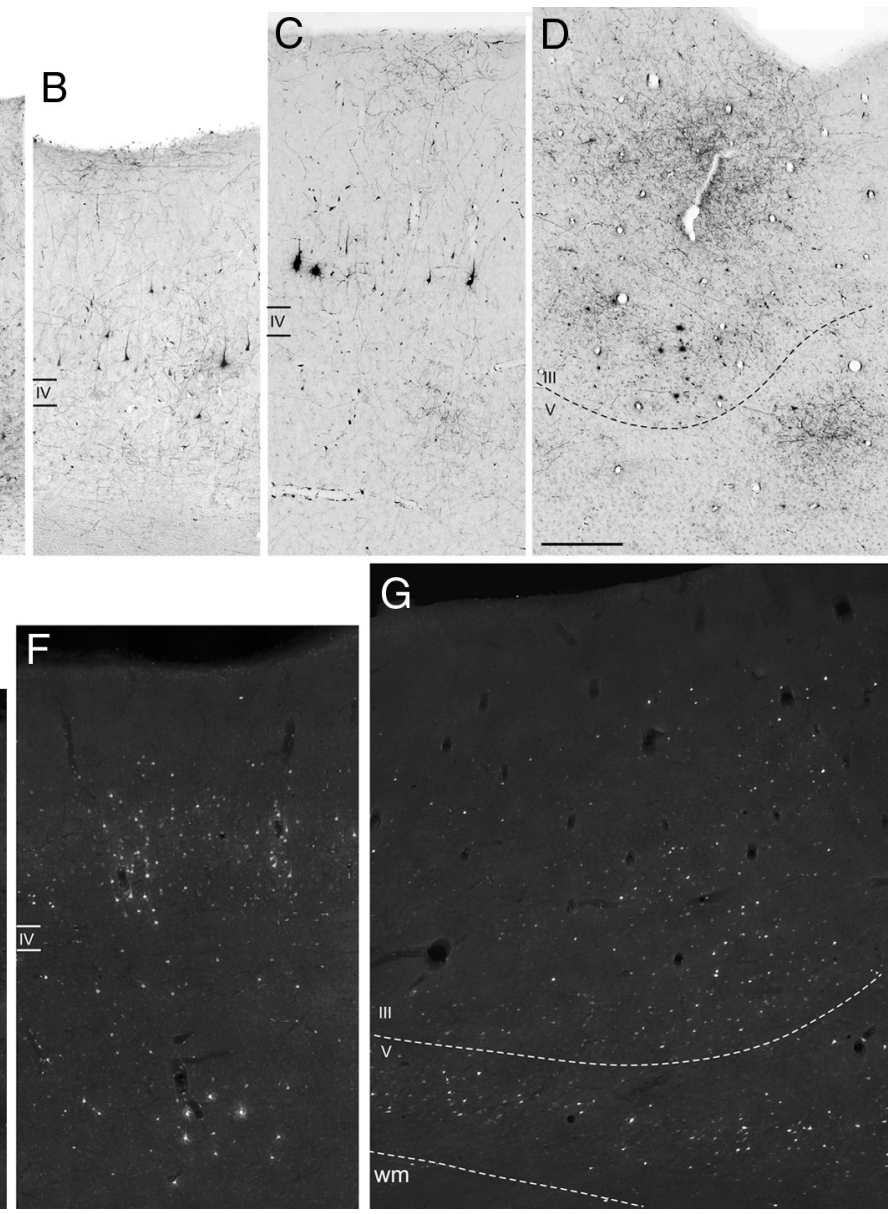

Figure 6. Examples of laminar patterns of retrograde and anterograde labeling, observed after injections in intermediate area 12 r, in areas $11(\boldsymbol{A}), \mathrm{TEa} / \mathrm{m}(\boldsymbol{B}, \boldsymbol{E}), \mathrm{SII}(\boldsymbol{C}, \boldsymbol{F})$, and F5a $(\boldsymbol{D}, \boldsymbol{G})$. $\boldsymbol{A}$ and $\boldsymbol{B}$ are from Case $44 \mathrm{r} F R, \boldsymbol{C}$ and $\boldsymbol{D}$ from Case 44r LYD, and $\boldsymbol{E}-\boldsymbol{G}$ from Case 43I FB. Scale bars: $\boldsymbol{A}, \boldsymbol{D}, 500 \mu \mathrm{m}(\boldsymbol{A}$ also applies to $\boldsymbol{B}, \boldsymbol{C}, \boldsymbol{E}-\boldsymbol{G})$. wm, White matter.

spectively. In Case 47r DY, relatively dense labeling was found in area F5a.

The injection site in Case 471 LYD, which was placed at $\sim 8$ $\mathrm{mm}$ rostral to the caudal tip of the PS, showed a distribution of retro-anterograde labeling compatible with a transition between the connectivity patterns displayed by the intermediate and caudal $12 \mathrm{r}$. Specifically, in this case, as after the injections were placed in intermediate 12r, there was labeling in F5a and AIP, the SII region, and the dysgranular insula. However, just as after injections in caudal 12r, the labeling in the orbitofrontal cortex was relatively weak and mostly confined to area $12 \mathrm{o}$ and in area $\mathrm{TEa} / \mathrm{m}$ extended more caudally than the labeling observed after the injections were placed in intermediate area $12 \mathrm{r}$.

The data from the "transitional" injections suggest that the changes in the connectional features of the caudal, intermediate, and rostral parts of area $12 \mathrm{r}$ do not occur homogenously.

\section{Discussion}

In the present study, we found that the macaque area $12 \mathrm{r}$ is connectionally not homogeneous and the intermediate part is fully integrated in a cortical network for selecting and controlling goaldirected object-oriented actions.

\section{Cortical connections of area $\mathbf{1 2 r}$}

Our results, summarized in Figure 10, provide evidence of three connectionally distinct zones located at different rostrocaudal 

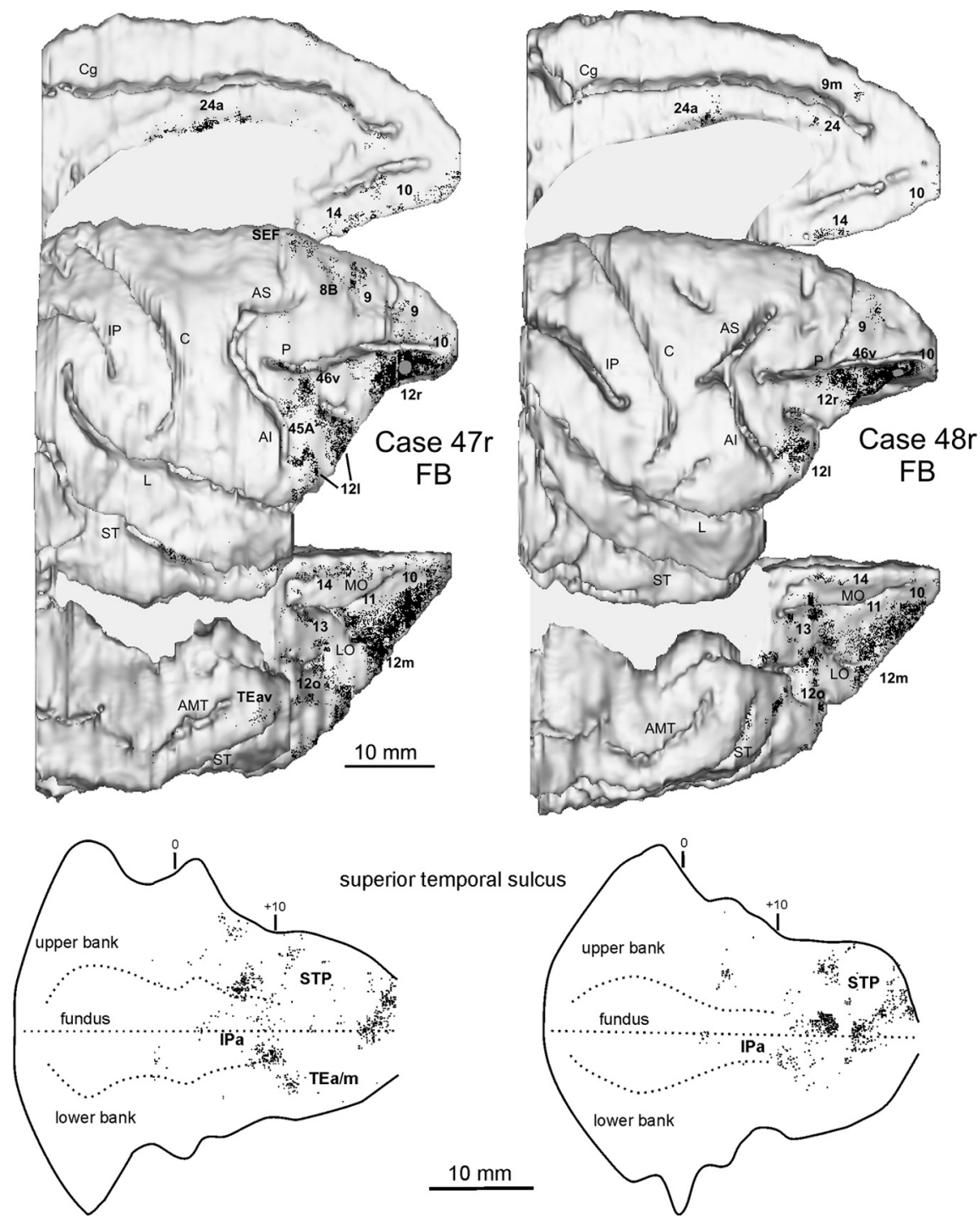

Figure 7. Distribution of the retrograde labeling observed after injections in rostral area $12 \mathrm{r}$ in Cases 47r FB and 48r FB, shown in dorsolateral, medial, and bottom views of the $3 \mathrm{D}$ reconstructions of the injected hemispheres (top) and in 2D reconstructions of the STS (bottom). Conventions and abbreviations as in Figures 1 and 3.

levels of area 12r. Specifically, we found that an intermediate zone of area $12 \mathrm{r}$, densely connected with area $46 \mathrm{v}$ and several orbitofrontal areas, is characterized by relatively strong extraprefrontal connectivity with ventral premotor, inferior parietal, opercular parietal, and inferotemporal areas and with the dysgranular insula. In contrast, a more rostral zone, although sharing with the intermediate zone connections with several orbitofrontal areas, is characterized by strong connectivity with rostral prefrontal areas and relatively weak extraprefrontal connectivity virtually all limited to the rostral upper bank of the STS. Finally, a caudal zone (Gerbella et al., 2010) displays strong connectivity with caudal VLPF areas, including areas 8/FEF and 45B, relatively weak and restricted orbitofrontal connectivity, and extraprefrontal connections limited to inferotemporal areas.

In this respect, our data indicate that intermediate area $12 \mathrm{r}$ shares some common connectional features (e.g., connections with F5, AIP, and the SII region) with area 46v (Barbas, 1988; Gerbella et al., 2010). However, the much stronger and extensive area $46 \mathrm{v}$ connections with F5 and the IPL, the much weaker temporal connections, and the virtual lack of orbitofrontal connections markedly distinguish this area from intermediate area $12 \mathrm{r}$.
The present study primarily extends the few previous observations based on one (Saleem et al., 2008) or two (Barbas, 1988) tracer injections, which have described connections compatible with an involvement of the injection sites mostly of the caudal (Barbas, 1988) or intermediate (Barbas, 1988; Saleem et al., 2008) part of area $12 \mathrm{r}$.

Some available indirect evidence is in line with the connectional heterogeneity of area $12 \mathrm{r}$ found in the present study. First, after tracer injections in the location of area $\mathrm{TEa} / \mathrm{m}$ (Saleem et al., 2008) or in the inferotemporal convexity cortex (Webster et al., 1994; Saleem et al., 2008; Borra et al., 2010b), the prefrontal labeling involved the caudal and intermediate parts, but not the rostral part, of area 12r. Second, after injections in F5a (Gerbella et al., 2011) or AIP (Borra et al., 2008), the labeling in area $12 \mathrm{r}$ was confined to the intermediate part. Finally, after injections in area 8/FEF (Huerta et al., 1987; Stanton et al., 1993; Gerbella et al., 2010), the labeling in area $12 \mathrm{r}$ was confined to the caudal part.

\section{Functional considerations}

The lateral prefrontal cortex is a functionally heterogeneous region essential for different aspects of executive functions, i.e., optimizing behavioral performance when cognitive processes are required (Tanji and Hoshi, 2008).

Specifically, the VLPF region located ventral to area 46 and including area $12 \mathrm{r}$ hosts visual neurons tuned to the identity or features of objects (Wilson et al., 1993; Asaad et al., 1998) and is critically involved in behavioral tasks in which information about object identity plays a key role (Passingham, 1975; Mishkin and Manning, 1978; Wang et al., 2000). Researchers have proposed that nonspatial information in this VLPF domain is at the basis of working memory for objects and shapes (Wilson et al., 1993), comparison and judgment of memorized information (Petrides, 2005), conditional learning based on object identity (Passingham, 1993; Passingham et al., 2000), and encoding category membership (Freedman et al., 2002; Miller et al., 2002) and abstract rules (Wallis et al., 2001).

To our knowledge, the functional properties of the entire extent of area $12 \mathrm{r}$ have not yet been systematically investigated. The only functional evidence for a possible heterogeneity of this region, has been provided by Hoshi et al. (2000), which described a tendency of shape- and location-selective neurons to cluster more rostrally and more caudally, respectively, likely at the level of intermediate and caudal $12 \mathrm{r}$.

The present data suggest that, in intermediate area $12 \mathrm{r}$, nonspatial information processing can be used for controlling goaldirected object-oriented hand and, possibly, mouth actions. First, intermediate area $12 \mathrm{r}$ is connected densely with F5 and, more weakly, with AIP, two tightly connected areas (Borra et al., 


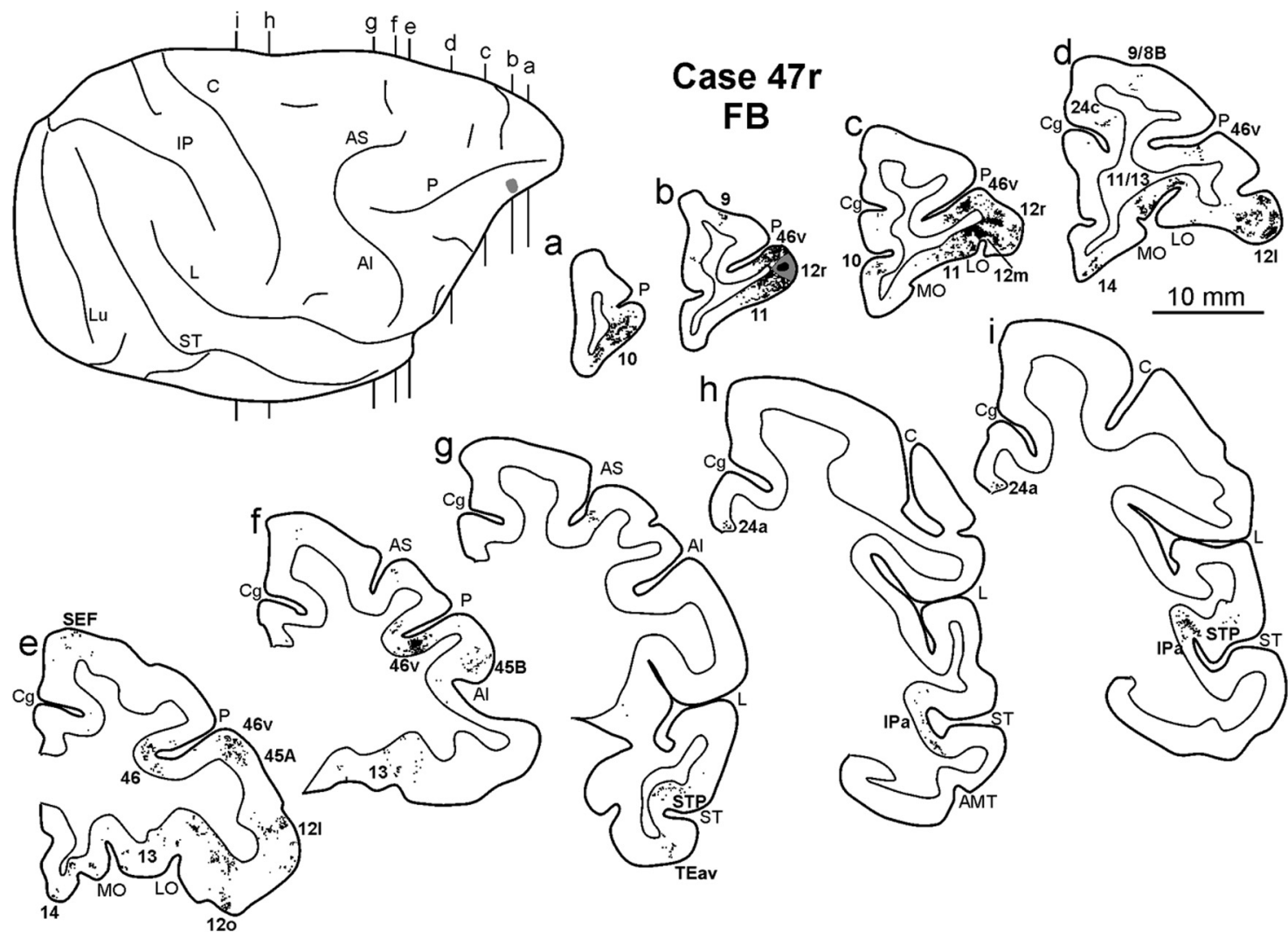

Figure 8. Distribution of the retrograde labeling observed in Case 47r FB, shown in drawing of coronal sections. Sections are shown in a rostral to caudal order (a-i). The dorsolateral view of the injected hemisphere in the upper left part of the figure shows the levels at which the sections were taken and the location of the injection site. Conventions and abbreviations as in Figures 1 and 3.

2008; Gerbella et al., 2011) forming a parietofrontal circuit mediating visuomotor transformations for grasping (Jeannerod et al., 1995; Rizzolatti and Luppino, 2001; Grafton, 2010). Specifically, the premotor connections of intermediate area $12 \mathrm{r}$ involve F5a, a subdivision of F5 that is a possible site of integration of parietal sensorimotor signals with higher-order information, originating from prefrontal and rostral frontal opercular areas, and F6/pre-supplementary motor area (Gerbella et al., 2011). The functional properties of F5a have not yet been systematically investigated. The connections with hand-related ventral premotor (F5p) and inferior parietal (AIP, PFG) fields suggest an involvement of F5a in controlling hand actions. Indeed, Fluet et al. (2010) recorded grasping neurons along a large extent of the postarcuate bank, very likely also in the location of F5a. Furthermore, functional imaging data (Nelissen and Vanduffel, 2011) show significant grasping-related activation in F5a. However, the connections with the lateral part of $\mathrm{F} 5 \mathrm{c}$ and the inferior parietal area PF suggest that mouth actions are also represented in F5a.

Second, intermediate area $12 \mathrm{r}$ is densely connected to a sector of SII, likely involving the hand and face/mouth fields, which appears to essentially overlap to the sector tightly linked to both AIP and F5, including F5a (Borra et al., 2008; Gerbella et al., 2011). The SII region is a higher-order somatosensory area, involved in haptic coding of objects (Reed et al., 2004) and tactile expectancies (Carlsson et al., 2000), and is a possible source of somatosensory information used by parietal and premotor areas for monitoring and updating motor programs.

Third, intermediate area $12 \mathrm{r}$ is densely connected to a sector of area $\mathrm{TEa} / \mathrm{m}$, which essentially overlaps with the $\mathrm{TEa} / \mathrm{m}$ sector strongly connected to AIP (Borra et al., 2008). This inferotempo- ral sector is a possible source of visual information related to object identity used by the AIP/F5 circuit for selecting the most appropriate grasping configurations as a function of the specific target object.

Finally, intermediate area $12 \mathrm{r}$ is connected to a sector of the dysgranular insula and opercular frontal areas whose functional properties are virtually unknown, but, being connected to AIP and F5 (Borra et al., 2008; Gerbella et al., 2011), appear to be likely involved in a cortical network for controlling hand actions.

The possible contribution of intermediate area $12 \mathrm{r}$ to the control of goal-directed actions still remains to be assessed. One possible working hypothesis is that intermediate area $12 \mathrm{r}$ contributes to the exploitation of nonspatial memory-based or working memory information related to object properties (e.g., weight, center of mass, fragility, texture) or identity, for controlling object-oriented actions. This information, based on implicit (sensorimotor memories) or explicit knowledge, could be used for grasping selection and anticipatory force control or for updating motor programs (Johansson and Cole, 1992; Lukos et al., 2007, 2008; Loh et al., 2010). Intermediate area $12 \mathrm{r}$ could also contribute to tactile object recognition, which requires retrieving memorized information about object properties and identity. Indeed, functional imaging studies in humans showed that tactile object recognition activates a cortical network that includes VLPF areas, in addition to temporal, inferior, and opercular parietal, and ventral premotor areas (Reed et al., 2004; Lacey et al., 2010; Savini et al., 2010). Furthermore, the dense connections with the orbitofrontal areas suggest that, in intermediate area $12 \mathrm{r}$, nonspatial information processing reflects affective properties or the "value" of objects (Schultz, 2000). This information could be 

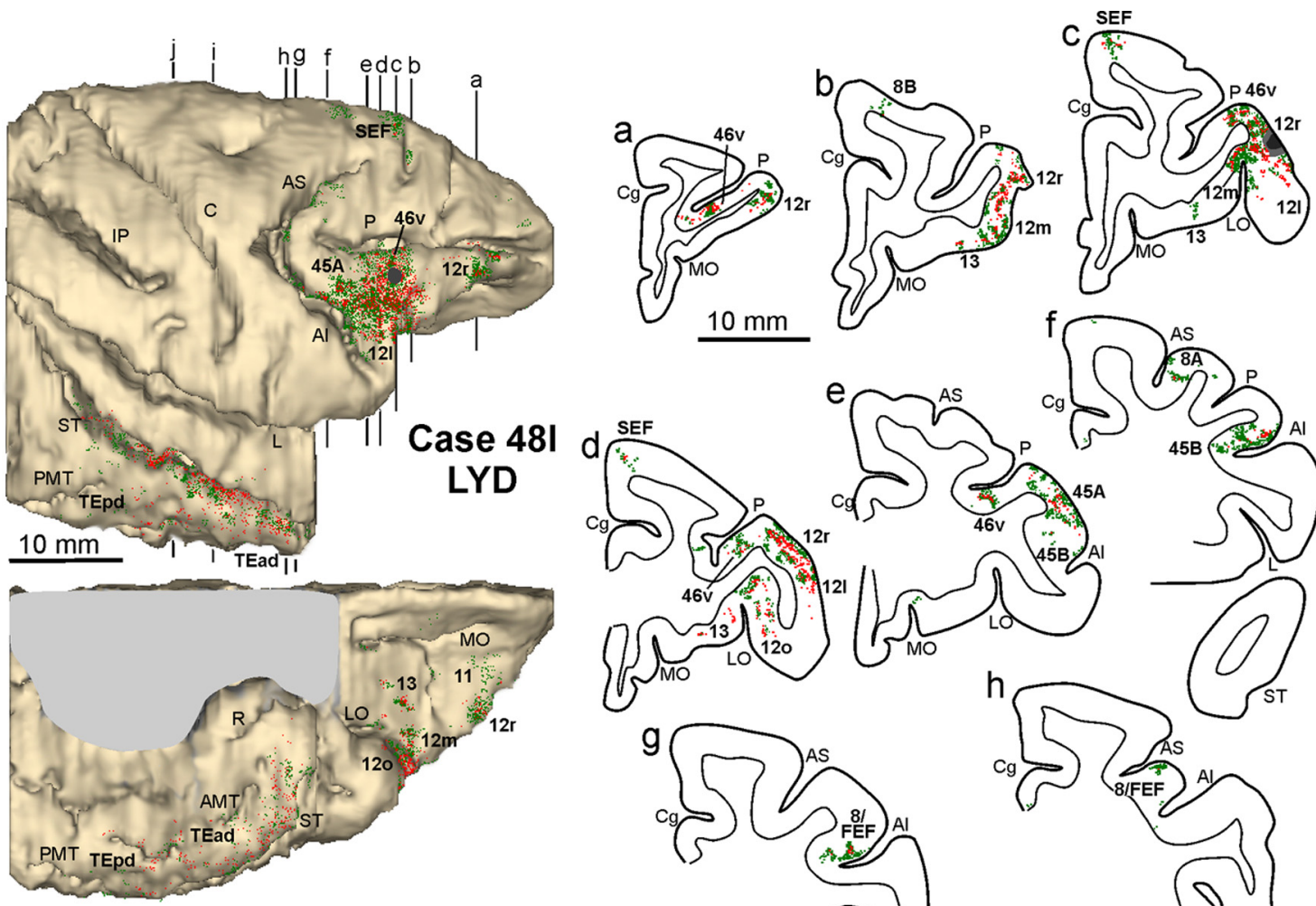

d

$10 \mathrm{~mm}$
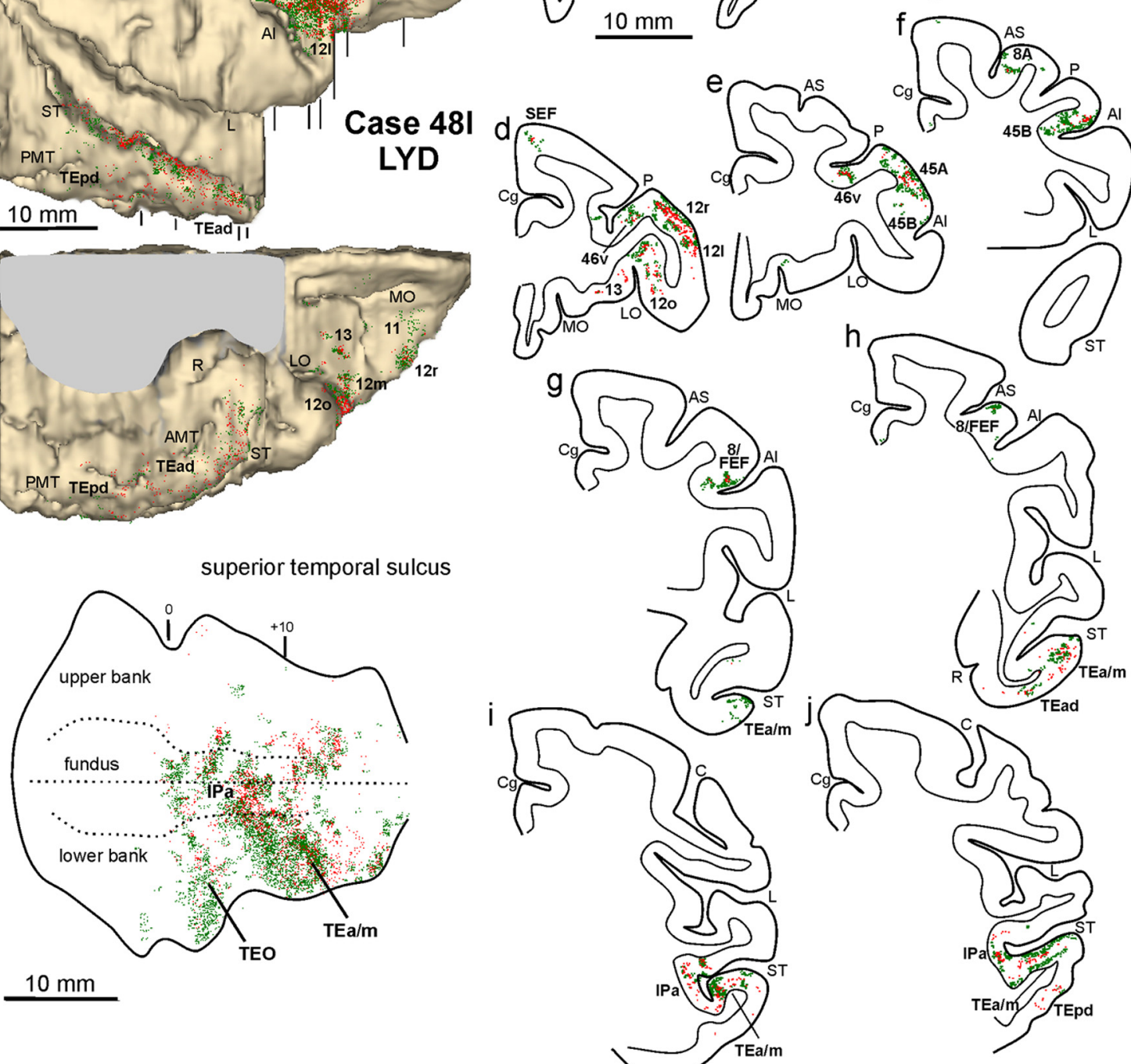

superior temporal sulcus 


\section{References}

Asaad WF, Rainer G, Miller EK (1998) Neural activity in the primate prefrontal cortex during associative learning. Neuron 21:1399-1407.

Barbas H (1988) Anatomic organization of basoventral and mediodorsal visual recipient prefrontal regions in the rhesus monkey. J Comp Neurol 276:313-342.

Barbas H, Rempel-Clower N (1997) Cortical structure predicts the pattern of corticocortical connections. Cereb Cortex 7:635-646.

Belmalih A, Borra E, Contini M, Gerbella M, Rozzi S, Luppino G (2009) Multimodal architectonic subdivision of the rostral part (area F5) of the macaque ventral premotor cortex. J Comp Neurol 512:183-217.

Bettio F, Demelio S, Gobbetti E, Luppino G, Matelli M (2001) Interactive 3-D reconstruction and visualization of primates cerebral cortex. Soc Neurosci Abstr 27:728.724.

Borra E, Belmalih A, Calzavara R, Gerbella M, Murata A, Rozzi S, Luppino G (2008) Cortical connections of the macaque anterior intraparietal (AIP) area. Cereb Cortex 18:1094-1111.

Borra E, Gerbella M, Rozzi S, Luppino G (2010a) Cortical connections of the ventrolateral prefrontal area 12r of the macaque monkey. Soc Neurosci Abstr 36:201.5

Borra E, Ichinohe N, Sato T, Tanifuji M, Rockland KS (2010b) Cortical connections to area TE in monkey: hybrid modular and distributed organization. Cereb Cortex 20:257-270.

Carlsson K, Petrovic P, Skare S, Petersson KM, Ingvar M (2000) Tickling expectations: neural processing in anticipation of a sensory stimulus. J Cogn Neurosci 12:691-703.

Carmichael ST, Price JL (1994) Architectonic subdivision of the orbital and medial prefrontal cortex in the macaque monkey. J Comp Neurol 346:366-402.

Condé F (1987) Further studies on the use of the fluorescent tracers fast blue and diamidino yellow: effective uptake area and cellular storage sites. J Neurosci Methods 21:31-43.

Felleman DJ, Van Essen DC (1991) Distributed hierarchical processing in primate cerebral cortex. Cereb Cortex 1:1-47.

Fitzgerald PJ, Lane JW, Thakur PH, Hsiao SS (2004) Receptive field properties of the macaque second somatosensory cortex: evidence for multiple functional representations. J Neurosci 24:11193-11204.

Fluet MC, Baumann MA, Scherberger H (2010) Context-specific grasp movement representation in macaque ventral premotor cortex. J Neurosci 30:15175-15184.

Freedman DJ, Riesenhuber M, Poggio T, Miller EK (2002) Visual categorization and the primate prefrontal cortex: neurophysiology and behavior. J Neurophysiol 88:929-941.

Gerbella M, Belmalih A, Borra E, Rozzi S, Luppino G (2007) Multimodal architectonic subdivision of the caudal ventrolateral prefrontal cortex of the macaque monkey. Brain Struct Funct 212:269-301.

Gerbella M, Belmalih A, Borra E, Rozzi S, Luppino G (2010) Cortical connections of the macaque caudal ventrolateral prefrontal areas $45 \mathrm{~A}$ and 45B. Cereb Cortex 20:141-168.

Gerbella M, Belmalih A, Borra E, Rozzi S, Luppino G (2011) Cortical connections of the anterior (F5a) subdivision of the macaque ventral premotor area F5. Brain Struct Funct 216:43-65.

Grafton ST (2010) The cognitive neuroscience of prehension: recent developments. Exp Brain Res 204:475-491.

Gregoriou GG, Borra E, Matelli M, Luppino G (2006) Architectonic organization of the inferior parietal convexity of the macaque monkey. J Comp Neurol 496:422-451.
Hoshi E, Shima K, Tanji J (2000) Neuronal activity in the primate prefrontal cortex in the process of motor selection based on two behavioral rules. J Neurophysiol 83:2355-2373.

Huerta MF, Krubitzer LA, Kaas JH (1987) Frontal eye field as defined by intracortical microstimulation in squirrel monkeys, owl monkeys, and macaque monkeys. II. Cortical connections. J Comp Neurol 265:332-361.

Jeannerod M, Arbib MA, Rizzolatti G, Sakata H (1995) Grasping objects: the cortical mechanisms of visuomotor transformation. Trends Neurosci 18:314-320.

Johansson RS, Cole KJ (1992) Sensory-motor coordination during grasping and manipulative actions. Curr Opin Neurobiol 2:815-823.

Kuypers HG, Huisman AM (1984) Fluorescent neuronal tracers. Adv Cell Neurobiol 5:307-340.

Lacey S, Flueckiger P, Stilla R, Lava M, Sathian K (2010) Object familiarity modulates the relationship between visual object imagery and haptic shape perception. Neuroimage 49:1977-1990.

Levy R, Goldman-Rakic PS (2000) Segregation of working memory functions within the dorsolateral prefrontal cortex. Exp Brain Res 133:23-32.

Loh MN, Kirsch L, Rothwell JC, Lemon RN, Davare M (2010) Information about the weight of grasped objects from vision and internal models interacts within the primary motor cortex. J Neurosci 30:6984-6990.

Lukos J, Ansuini C, Santello M (2007) Choice of contact points during mul- 
tidigit grasping: effect of predictability of object center of mass location. J Neurosci 27:3894-3903.

Lukos JR, Ansuini C, Santello M (2008) Anticipatory control of grasping: independence of sensorimotor memories for kinematics and kinetics. J Neurosci 28:12765-12774.

Luppino G, Calzavara R, Rozzi S, Matelli M (2001) Projections from the superior temporal sulcus to the agranular frontal cortex in the macaque. Eur J Neurosci 14:1035-1040.

Luppino G, Rozzi S, Calzavara R, Matelli M (2003) Prefrontal and agranular cingulate projections to the dorsal premotor areas F2 and F7 in the macaque monkey. Eur J Neurosci 17:559-578.

Matelli M, Luppino G, Rizzolatti G (1985) Patterns of cytochrome oxidase activity in the frontal agranular cortex of macaque monkey. Behav Brain Res 18:125-136.

Matelli M, Luppino G, Rizzolatti G (1991) Architecture of superior and mesial area 6 and the adjacent cingulate cortex in the macaque monkey. J Comp Neurol 311:445-462.

Matelli M, Govoni P, Galletti C, Kutz DF, Luppino G (1998) Superior area 6 afferents from the superior parietal lobule in the macaque monkey. J Comp Neurol 402:327-352.

Medalla M, Barbas H (2006) Diversity of laminar connections linking periarcuate and lateral intraparietal areas depends on cortical structure. Eur J Neurosci 23:161-179.

Miller EK, Freedman DJ, Wallis JD (2002) The prefrontal cortex: categories, concepts and cognition. Philos Trans R Soc Lond B Biol Sci 357:1123-1136.

Mishkin M, Manning FJ (1978) Non-spatial memory after selective prefrontal lesions in monkeys. Brain Res 143:313-323.

Nelissen K, Vanduffel W (2011) Grasping-related functional magnetic resonance imaging brain responses in the macaque monkey. J Neurosci 31:8220-8229.

Nelissen K, Borra E, Gerbella M, Rozzi S, Luppino G, Vanduffel W, Rizzolatti G, Orban GA (2011) Action observation circuits in the macaque monkey cortex. J Neurosci 31:3743-3756.

Passingham R (1975) Delayed matching after selective prefrontal lesions in monkeys (Macaca mulatta). Brain Res 92:89-102.

Passingham RE (1993) The frontal lobe and voluntary action. Oxford: Oxford UP.

Passingham RE, Toni I, Rushworth MF (2000) Specialisation within the prefrontal cortex: the ventral prefrontal cortex and associative learning. Exp Brain Res 133:103-113.

Petrides M (2005) Lateral prefrontal cortex: architectonic and functional organization. Philos Trans R Soc Lond B Biol Sci 360:781-795.

Petrides M, Pandya DN (2002) Comparative cytoarchitectonic analysis of the human and the macaque ventrolateral prefrontal cortex and corticocortical connection patterns in the monkey. Eur J Neurosci 16:291-310.
Reed CL, Shoham S, Halgren E (2004) Neural substrates of tactile object recognition: an fMRI study. Hum Brain Mapp 21:236-246.

Rempel-Clower NL, Barbas H (2000) The laminar pattern of connections between prefrontal and anterior temporal cortices in the rhesus monkey is related to cortical structure and function. Cereb Cortex 10:851-865.

Rizzolatti G, Craighero L (2004) The mirror neuron system. Annu Rev Neurosci 27:169-192.

Rizzolatti G, Luppino G (2001) The cortical motor system. Neuron 31:889-901.

Rozzi S, Calzavara R, Belmalih A, Borra E, Gregoriou GG, Matelli M, Luppino G (2006) Cortical connections of the inferior parietal cortical convexity of the macaque monkey. Cereb Cortex 16:1389-1417.

Saleem KS, Tanaka K (1996) Divergent projections from the anterior inferotemporal area TE to the perirhinal and entorhinal cortices in the macaque monkey. J Neurosci 16:4757-4775.

Saleem KS, Kondo H, Price JL (2008) Complementary circuits connecting the orbital and medial prefrontal networks with the temporal, insular, and opercular cortex in the macaque monkey. J Comp Neurol 506:659-693.

Savini N, Babiloni C, Brunetti M, Caulo M, Del Gratta C, Perrucci MG, Rossini PM, Romani GL, Ferretti A (2010) Passive tactile recognition of geometrical shape in humans: an fMRI study. Brain Res Bull 83:223-231.

Schultz W (2000) Multiple reward signals in the brain. Nat Rev Neurosci 1:199-207.

Seltzer B, Pandya DN (1978) Afferent cortical connections and architectonics of the superior temporal sulcus and surrounding cortex in the rhesus monkey. Brain Res 149:1-24.

Stanton GB, Bruce CJ, Goldberg ME (1993) Topography of projections to the frontal lobe from the macaque frontal eye fields. J Comp Neurol 330:286-301.

Tanji J, Hoshi E (2008) Role of the lateral prefrontal cortex in executive behavioral control. Physiol Rev 88:37-57.

Walker EA (1940) A cytoarchitectural study of the prefrontal area of the macaque monkey. J Comp Neurol 73:59-86.

Wallis JD, Anderson KC, Miller EK (2001) Single neurons in prefrontal cortex encode abstract rules. Nature 411:953-956.

Wang M, Zhang H, Li BM (2000) Deficit in conditional visuomotor learning by local infusion of bicuculline into the ventral prefrontal cortex in monkeys. Eur J Neurosci 12:3787-3796.

Webster MJ, Bachevalier J, Ungerleider LG (1994) Connections of inferior temporal areas TEO and TE with parietal and frontal cortex in macaque monkeys. Cereb Cortex 4:470-483.

Wilson FA, Scalaidhe SP, Goldman-Rakic PS (1993) Dissociation of object and spatial processing domains in primate prefrontal cortex. Science 260 : 1955-1958. 